\title{
RECURRENT VISION WITH A MOVING STIMULUS OF ALTERNATING INTENSITY*
}

\author{
BY \\ F. L. Warburton, M.Sc. \\ BARNATO JOEL LABORATORIES, MIDDLESEX HOSPITAL
}

THE temporal course of the visual sensation produced by a brief stimulus has been observed by causing a bright image to travel across the retina in a circular path. Observations have been made with a stimulus of alternating intensity and also with one of constant intensity. Various speeds of traverse were used, and also varying intensities and conditions of dark adaptation.

The moving stimulus was obtained by rotating an opaque disc, which had a radial slot cut in it, in front of an illuminated sheet of opal glass. The glass was illuminated by an electric lamp placed in a large white box of which the glass formed one side. By this means a very high intensity of illumination was obtained. Alternating intensity of illumination of 100 cycles frequency was obtained by supplying the lamp with 50 cycle alternating current. Constant intensity was obtained by running the lamp off batteries. When a large lamp was used to obtain high intensities, the variation in intensity was much smaller owing to the increased thermal lag. A red lamp was provided in a position inside the circle to assist in keeping the eye steady and to ensure that extrafoveal vision was used.

When the disc revolved at slow speed (about 2 revs. per second), a broad bright band was seen, which with a coloured stimulus (obtained by placing a filter over the slot) was the same colour as the stimulus. This band moved round with the disc. Using alternating intensity this band was divided into light and dark radial strips which remained stationary and did not move round with the disc. The bright band was followed by a dark band and then by another bright band, which also moved round with the disc. The latter band was divided into light and dark stationary strips, like the first bright band. When the stimulus was coloured the second band showed the complementary colour. With high intensities the second band was very broad and faded away slowly. With lower intensities it was narrower and with the lowest intensities used it was narrower than the primary band.

The dark band was in general blacker than the surrounding unilluminated field; it was thus probably due in part to temporal induction from the preceding bright band, as any effect due to spatial induction would be present in the immediately adjacent portions of the surrounding field:

- Aided by a grant from the Medical Research Council. 
When the speed of the disc was increased the first moving band widened out until it filled nearly the whole circle; the angular distance between the stationary strips was also increased, but the bright band covered a larger number of these strips. The second moving band was also broadened out and usually overlapped the primary band as the latter followed round for a second time. In some cases the second band seemed to move round on top of the primary. It was usually narrower than the primary band as the total amount of light falling on the retina was comparatively low owing to the much shorter time during which the retina was exposed. The phenomena with the increased speed were much more clearly observed with coloured stimuli owing to the difference in colour between the primary and secondary bands.

Using a stimulus of constant intensity a similar appearance was produced except that the stationary strips were absent. The second bright band always showed a gradually diminishing intensity, whereas the first usually showed slight radial variations in intensity. These lighter and darker strips moved round with the disc, instead of being stationary, as were the much more pronounced light and dark strips seen with an alternating stimulus. They were thus purely subjective and not due to stimulus variations as were the latter. They should therefore also be present when an alternating stimulus is used, although they would tend to be masked by the stimulus strips, particularly with low intensities. Their existence was, however, shown by an unpleasant flickering seen in the primary band, which in some cases could be recognized as due to the disappearance of different bright strips. This occurred when a bright part of the stimulus variation coincided with a dark portion of the subjective variation. In general this would only occur for one strip at a time, owing to the different angular separation of the two phenomena. The effect was seen best with a coloured stimulus, being most pronounced for green.

The phenomena observed with a stimulus of constant intensity have been studied by various workers, ${ }^{1}$ and are due to complicated physiological processes in the retino-cerebral apparatus.

The explanation usually given is that, under the conditions of observation, a momentary stimulus produces a brief pulsating sensation followed by a dark interval which in its turn is followed by another less bright sensation of gradually diminishing intensity. With coloured stimuli the second sensation shaws the complementary colour. (These phenomena were all observed by looking away from the slit and momentarily switching on the light).

With a moving stimulus this sequence is produced in every part of its path, so that adjacent parts of the retina show successive stages in the sequence. This will also apply when the stimulus 
is of alternating intensity and the disappearance of one or more stationary strips will occur when the sensation produced, at a spot on the retina where the stimulus was at maximum intensity, reaches one of its minima. The sensation produced in adjacent parts of the retina, where the stimulus is at its minimum intensity, will then be approximately at maximum intensity; there will thus be very little difference of intensity over an area corresponding to the distance between two alternate bright strips. In general only one bright strip will disappear in this way at any given instant.

All the phenomena observed receive a satisfactory explanation on the basis of the general form of the hypothesis usually adopted to account for the phenomena observed when a moving stimulus of constant intensity travels across the retina. They thus support the hypothesis that these phenomena are largely due to the temporal course of the sensation produced by a stimulus of short duration, although spatial induction may inhibit some part of the course of sensation. This latter factor would appear to act with alternating intensity, and so to be due to the whole of the bright part of sensation, and not merely to that in parts of the retina immediately adjacent to that where inhibition took place.

The unpleasant flickering is not to be confused with that observed when a narrow object (such as a billiard cue), illuminated by an A. C. lamp, is rapidly moved to and fro laterally. This is due entirely to light and dark strips seen in the primary band. It may, however, contribute to the nervous irritation sometimes produced under these conditions, or which some people find (probably due to eye movements) with A. C. lighting under ordinary conditions.

\section{REFERENCE}

1. MacDougall, Brit. Jl. of Pysch., Vol. I, 1904; NAgel, Handbuch der Physiol. des Menschen; PARsons, Colour Vision, London, 1924.

\section{ROYAL LONDON OPHTHALMIC HOSPITAL (Moorfields)}

IN connection with the opening of the new Lecture Hall on October 1 , the following has been contributed by a correspondent.

The Out-Patient Department, Library, Lecture Room, Museum, and Pathological Laboratory were erected in 1898. The Bacteriological Laboratory was added in 1907. In 1925 Sir William Lister built and equipped a smaller lecture room for clinical demonstrations. 